\title{
INTEROPERABLE INFORMATION MODEL OF A PNEUMATIC HANDLING SYSTEM FOR PLUG-AND-PRODUCE
}

\author{
Raphael Alt ${ }^{1 *}$, Peter Wintersohle ${ }^{1}$, Hartmut Schweizer ${ }^{2}$, \\ Martin Wollschlaeger ${ }^{2}$, Katharina Schmitz ${ }^{1}$ \\ 1: Institute for Fluid Power Drives and Systems, RWTH-Aachen University, Campus-Boulevard 30, 52074 Aachen \\ 2: Institute for Applied Computer Science, TU Dresden, Nöthnitzer Str. 43, 01187 Dresden \\ * Corresponding author: Tel.: +49 24180 47729; E-mail address: raphael.alt@ifas.rwth-aachen.de
}

\begin{abstract}
Commissioning of a machine is still representing a very challenging operation and most steps are still executed manually by commissioning engineers. A future goal is to support the commissioning engineers and further automate the entire integration process of a newly installed system with a minimum of manual effort. This use case is known as plug-and-produce (PnP). In this contribution a concept of the Industrial Internet of Things is presented to improve the commissioning task for a pneumatic handling system. The system is based on a service-oriented architecture. Within this context, information models are developed to meet the requirements of $\mathrm{PnP}$ to provide relevant information via virtual representations, e.g. the asset administration shell, of the components to the commissioning process. Finally, a draft of the entire PnP process is shown, providing a general understanding of Industrial Internet of Things fluid power systems.
\end{abstract}

Keywords: IIoT, Plug-and-Produce, Pneumatic, Interoperability, Industry 4.0, Service-oriented architecture, Orchestration, Commissioning

\section{INTRODUCTION}

"Industrie 4.0" (I4.0) represents an initiative, introduced by the German government to maintain the competitiveness of the manufacturing industry in 2011 [1]. In this context, classical production systems are enhanced by new Information and Communication Technologies (ICT) to increase the productivity, flexibility and efficiency as well as enabling new digital and thus scalable business models to raise the profitability of the manufacturing sector [2]. Internationally, similar concepts are known under the expression Industrial Internet of Things (IIoT). [3]

Pneumatic fluid power systems state an important role in many automation lines. Handling and Pick-and-Place systems for example, which are usually built-in in an automation line, can contain several pneumatic rotational and linear actuators as well as grippers. The commissioning process of such automation lines, which includes the integration of the pneumatic system, is still posing a challenging operation. For the first time in a product life cycle, various subsystems of different domains and manufacturers need to work together in a machine. Due to the vast variety of different system configurations and the occurrence of unforeseen events, the commissioning represents an error-prone and complex task that is mostly executed manually by specialized commissioning engineers (CE) today. This makes the process time-consuming and costly. $[4,5]$

Increasing international competition and changing market trends require to reconsider the requirements of commissioning with regard to economical and technical aspects. The current trends, like manufacturing individual products at low costs (mass customization), shortening of product life cycles, demands for effective maintenance, higher functionality and higher reliability of machines, all result in an increasing complexity of automation lines as well as frequent reconfigurations to continuously meet the changing requirements $[3,6]$. Consequently, due to recurrent setups and reconfigurations of machines during lifetime, the economic importance of the commissioning process becomes more important in the product life cycle. 
Additionally, the rising complexity of the machines challenges and overwhelms the CE. Assistant systems can support the personnel to a certain point [7], but an almost entirely automated commissioning process states the desired goal to meet the upcoming market demands.

Such a system requires two elements. First and for most, it requires a framework to provide and process knowledge about the commissioning of the system in order to derive steps that need to be performed. The automated sequencing of tasks is known as orchestration and is based on a semantic and interoperable information structure for the pneumatic systems that can be interpreted by different ICTs. This aspect will be focused in this contribution. Secondly, the system requires actuators to perform the required commissioning steps to fully automate the process. Here, many different solutions, depending on the component can be realized by the manufacturers. [8]

In this contribution, an approach based on concepts of distributed systems (e.g. the internet) is developed and modified to fit industrial applications in order to support the commissioning of a pneumatic handling system. This use-case is known as "Plug-and-Play", "Plug-and-Produce" (PnP) or "Plug-and-Work" [4].

After presenting the commissioning of the pneumatic handling demonstrator, the main aspects are identified, which need to be reflected by an automated assistant system. Basic elements of an IIoT system are introduced in general. As one important element, a virtual representation, serving as an information model for the commissioning of the pneumatic system, is derived. Based on that, a draft for the orchestration process is given. Finally, the implementation at the demonstrator represents a prove of concept and provides a general understanding of the main aspects of IIoT systems and its implications to the automated integration of fluid power devices.

\section{COMMISSIONING OF A PNEUMATIC HANDLING SYSTEM}

A pneumatic reference system is used to analyze the commissioning. It consists of a pneumatic handling system, a valve terminal and a pressure supply. As an example pneumatic handling systems are used in industrial packaging systems.
Figure 1 depicts the pneumatic handling system in detail.

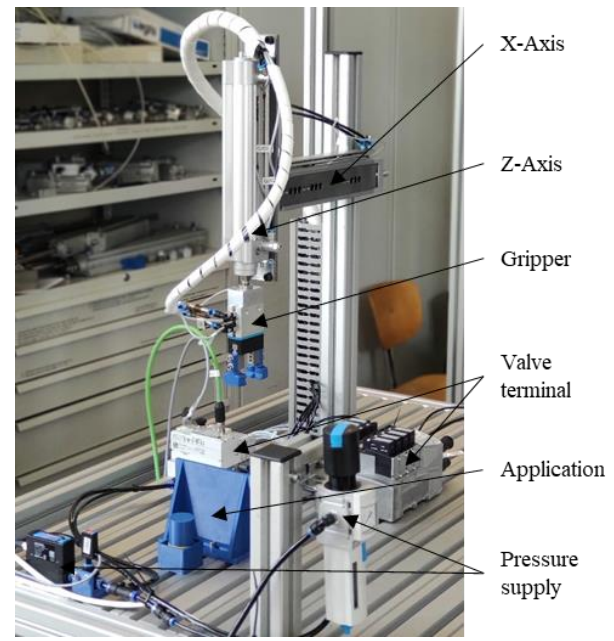

Figure 1: Pneumatic handling system

The pneumatic handling system consist of three pneumatic actuators: $\mathrm{x}$-axis, $\mathrm{z}$-axis and a gripper. Each axis consists of a pneumatic drive which can be further subdivided into a linear pneumatic cylinder and two end position sensors. The pressure supply consists of a constant pressure source, a pressure sensor, a mass flow sensor and a manually adjustable pressure regulating valve. The system is operated via a programmable logic controller. This controller provides the sensor signals and functions of all the installed system components.

The commissioning of pneumatic systems is a complicated process, consisting of different commissioning tasks, which start after the completion of its assembly. The assembling and the commissioning are often executed by different people. Consequently, the CE needs detailed information on the system such as a system overview, a pneumatic circuit diagram and individual datasheets of its components. The $\mathrm{CE}$ derives an individual commissioning procedure depending on the structure of the pneumatic system and its components. Furthermore, the CE needs to identify and solve problems that result from the assembly process and ones that occur unforeseen during the commissioning. Therefore, expert knowledge is required to safely conduct the commissioning process. 
Analysing the commissioning of the reference system shows that the commissioning process of a pneumatic systems can be divided into three sequential phases. During the first phase of commissioning the pneumatic system is unpressurised. All commissioning activities during this phase have the objective to put the system into a fail-safe state to avoid critical errors when the system gets pressurised later on. These activities include gathering information and visual inspection of the components. The pneumatic circuit scheme provides an overview of all components and their interactions. After checking all sensor signals for plausibility, the pneumatic end position dampers and throttle check-valves are adjusted according to the manufacturer specifications. The next phase begins via adjusting the pressure regulation valve of the power supply to an overall system minimum pressure level. The pressure level is chosen based on the required minimum pressure to safely operate relevant components to avoid severe collisions and damage resulting from uncontrolled movements of the system. All sensors are checked repeatedly to ensure valid values, followed by various tests regarding pneumatic functions. The tests include the switching of all valves and checking for the correct movements of the intended actuators and the sequence of the working cycle. The third commissioning phase starts by setting the system pressure to the intended operating level. In this phase, the cycle-times of the actuators are set via adjusting the throttle check-valves. After the pneumatic end position damping is tuned correctly, the system performs an entire working cycle to ensure correct interactions with other modules of the automation line. With the successful completion of all tasks, the system is commissioned and ready to operate. The analysis of the commissioning process shows that it can be time-consuming, prone to errors and thus specialist knowledge is indispensable.

Summarizing, the commissioning process can be divided into three different phases according to the pressure level: unpressurized, low pressure and operating pressure. This serves as a general frame for the CE or an automated system to sort specific system and component commissioning activities. To do so, both must be aware of all the required tasks, their correct sequential order and the corresponding relevant parameters.

\section{BASIC ELEMENTS OF IIOT SYSTEMS}

The concepts of the Internet of Things (IoT) in general as well as the IIoT in specific originate from the field of distributed systems. In contrast to classical systems, which are controlled via hierarchical and centralized patterns (e.g. automation pyramid), they consist of different collaborating assets to solve a task in a more flexible way. To do so, the individual assets offer encapsulated functionalities, which can easily be used by other system participants. The so-called services can then be combined to solve different system tasks. The process of combining services in such a service-oriented architecture (SOA) is called orchestration and represents a key element of IIoT systems.

The SOA leads to implications for the assets, as shown for a pneumatic component in figure 2 . The smart IIoT component consists of the real object, combining the former proprietary hardand firmware of the pneumatic asset. In addition, the real object is enhanced by the following aspects, building the virtual representation of the asset.

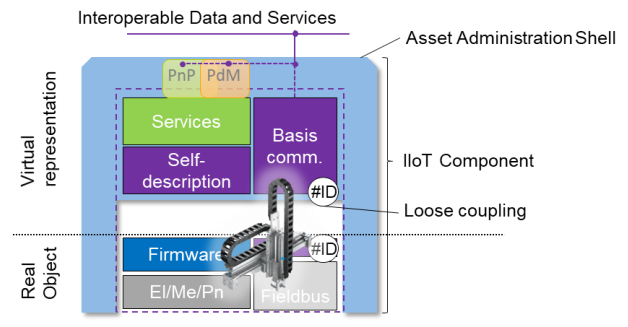

Figure 2: IIoT component using the AAS, after [3]

Services: Encapsulated functionalities of the asset to facilitate the usage for other system participants. Only inputs and outputs of the service interfaces are known and provided externally. The internal function remains untouched and resembles as a black box for the user.

Self-description: Part of an information model and provides data and information to other system participants about its identification, properties and functions. Additionally, it contains relevant aspects of different use-case scenarios, e.g. PnP or predictive maintenance (PdM).

Basic communication and interoperability: To collaborate system-wide, every IIoT component requires an open communication that is independent on proprietary and physical 
communication interfaces (e.g. OPC UA, MQTT). Additionally, the broadcasted data needs to be interpreted correctly by every other interacting system participant. Thus, standardization and semantic description of data and information enables system-wide interoperability.

The asset administration shell (AAS) represents a concept to combine the mentioned aspects of the virtual representation in order to enable a consistent interaction of all different kinds of assets and thus to integrate them into an IIoT system. [9, 10, 11]

\section{INFORMATION MODEL OF PNEUMATIC IIOT COMPONENT}

THE

The pneumatic IIoT component consists of the asset (physical pneumatic component) and the corresponding virtual representation, the asset administration shell (AAS). All relevant information and real-time data of the components are represented via properties within the AAS. Properties are grouped according to their objectives and assigned to a submodel. To ensure interoperability to a certain part, submodels and properties are currently defined by different industrial associations. [12]

Figure 3 shows a class diagram of the AAS for a pneumatic drive. The depicted class diagram focuses only relevant aspects for $\mathrm{PnP}$ of the corresponding asset. Submodels, which are relevant for other parts in the component's lifecycle, e.g. condition monitoring, can be added to the existing model.

The submodel 'AssetIdentification' includes properties to identify the asset like the asset identifier and other unique identifiers such as the manufacturers name and serial number. These properties are used to find the AAS inside a digital network. Properties that describe the technical aspects of the asset are assigned to the submodel 'Datasheet'. It resembles a standardized digital version of today's existing datasheets and can be used to further derive more information. For example, the cylinder's velocity can be derived using the cylinder's stroke and the switching-time of the end position switches. Properties of the operational phase are assigned to the submodel 'Operating'. In contrast to properties of the datasheet, they can vary with time and reflect the current state of the component. For example, the current cycle times of the drive can be measured and compared to the target cycle times, which were set beforehand during the engineering phase of the system.

The submodel 'Topology' contains properties to reflect different kinds of dependencies of the assets in the system. The different topologies can give functional, structural, fluidic, electric, business and other views on the system. In this case, a functional and a fluidic view are focused for the commissioning process. The fluid power topology describes the airflow through the pneumatic system similar to the pneumatic circuit

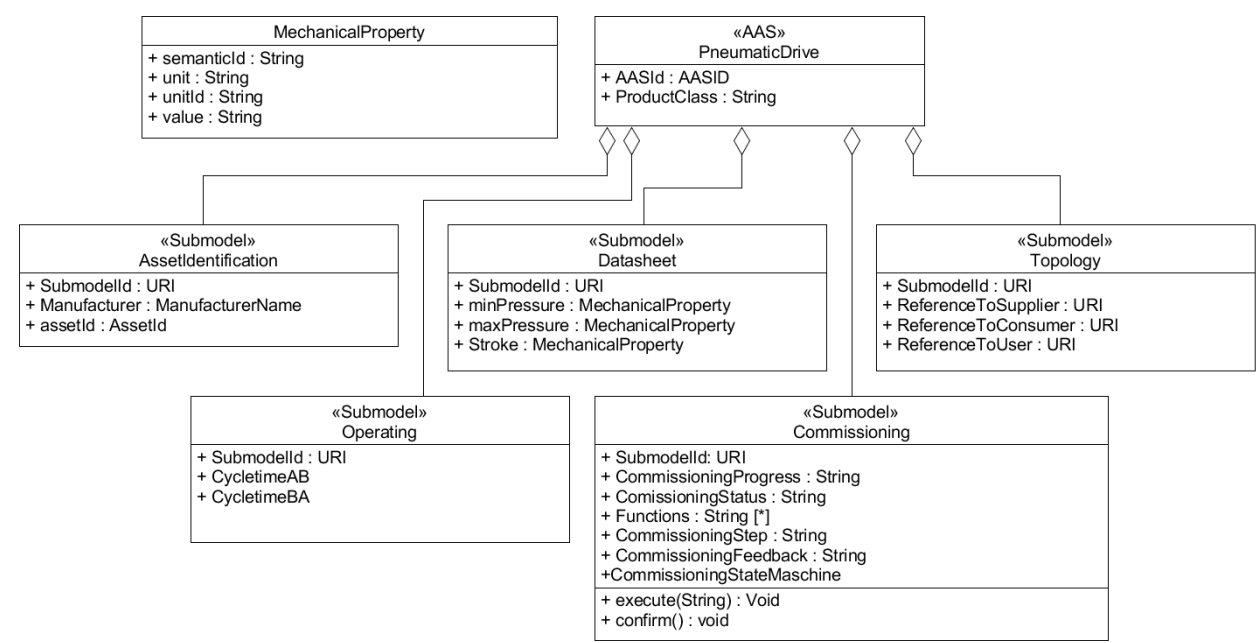

Figure 3: Class diagram asset administration shell pneumatic drive 
diagram. Within the properties 'ReferenceToSupplier' and 'ReferenceToConsumer' the up- and downstream connected pneumatic components can be referred.

Figure 4 shows the fluid power topology of the reference system. The functional topology describes functional interactions between the components which can be derived from the system control. System-wide topologies can be derived from different engineering documents which are created during the design phase of a system. Afterwards they are stored distributed in the information models of different AASs and can be used during the assembly, commissioning, operation or other phases. During PnP, both topologies are used to directly interact with other relevant AASs in order to exchange information on the current commissioning progress or use services.

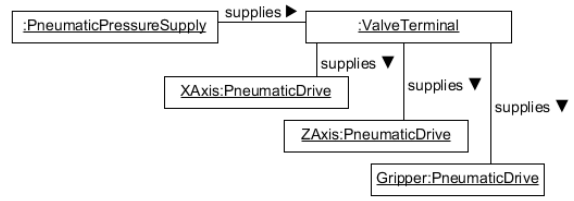

Figure 4: Fluid power topology of the pneumatic system

All other properties which are required for $\mathrm{PnP}$ and the corresponding orchestration are listed in the submodel 'Commissioning'. It includes all individual commissioning steps that need to be carried out, their preconditions and the available functionality of the assets.

To further detail the submodel for PnP, all previously analyzed commissioning activities are structured and grouped into commissioning steps. Each commissioning step has a precondition that determines when it can be carried out. It ends, when all necessary commissioning activities of this step are competed. Preconditions either refer to their own or to other component's PnP parameters, e.g. the commissioning progress. Commissioning steps and preconditions are implemented in a state machine. It is not visible to the outside world, thus not shown in the class diagram.

Figure 5 gives a brief overview of the state machine. Each state machine of a component has four generalized governing states INIT, Pre-Op, Safe-Op, Op, that indicate the progress of commissioning. Each state can contain additional component specific commissioning steps. Component specific error handling can also be implemented in each step. Depending on the sensors installed, each component can detect errors during commissioning and operation.

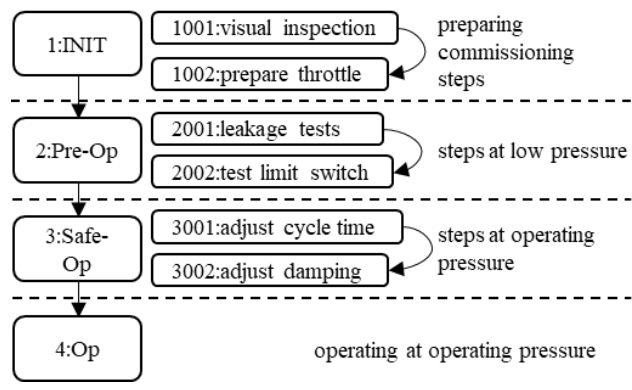

Figure 5: Diagram of the commissioning state machine inside an AAS

The AAS only exposes the next, respectively the currently running commissioning step within the property 'CommissioningStep'. A status property tells whether a commissioning step is currently running.

The property 'CommissioningFeedback' states a required precondition or an action that needs to be performed by other components in order to complete the running commissioning step. It is used to interact with other components or with the CE. The AAS gives out the required information or functionality. For example, a pneumatic drive requires the adjustment of enddamping.

The property 'Functions' lists functionalities that can be used by other components. This property is continuously updated, since the asset can provide additional functions as it increases its commissioning state towards OP state.

Two basic services are implemented in the AAS. 'Execute' is used to start a function or a commissioning step, 'confirm' is used to confirm that a requested function has been executed by another component or by the CE.

In the pneumatic reference system, the AASs are deployed on the PLC. Thus, a direct interaction between AAS and asset takes place. The AAS can directly access functions that are mapped in the control system and record realtime data of the asset. 


\section{PLUG-AND-PRODUCE SEQUENCE AT THE DEMONSTRATOR}

The PnP commissioning starts after the assembly of the pneumatic handling system. A PnP-module is used to orchestrate and monitor the automated commissioning process. The orchestration, monitoring and error handling is done based on a state machine inside the PnP-module. In addition, it is used to guide the $\mathrm{CE}$ through the commissioning process. All necessary information are displayed on a human machine interface (HMI). Buttons of the HMI are used to confirm error messages and manual adjustments that had been carried out by the engineer.

The module is implemented on a single board computer and can therefore be used to commission various systems. OPC Unified Architecture (OPC UA) is used for communication between the AASs and the PnPmodule.

Figure 6 shows a commissioning cycle of the PnP-module during the orchestration of the commissioning.

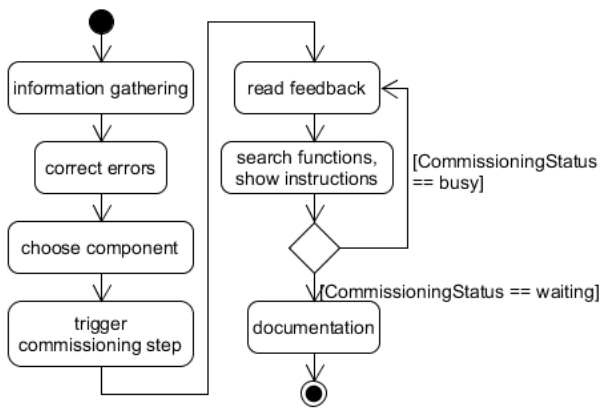

Figure 6: Activity diagram of a commissioning cycle during the orchestration of the PnP-module

Each cycle of the commissioning process starts with the collection of information. The PnPmodule reads properties of the commissioning submodel of each AAS. If an error occurs the PnP-module starts an error handling sequence, showing the error massage and a trouble shooting hint on the HMI. Then, the component and its commissioning step is chosen based on the collected information. After the commissioning is triggered, the PnP-module reads the feedback property. If the required commissioning step of a component exceeds its own capabilities, the PnPmodule searches for the appropriate functionality in the function property of the other AASs. The
$\mathrm{CE}$ is not excluded from this procedure and will additionally be consulted for backup if no other component can provide a suitable function. In this case the instructions will be displayed on the HMI. The CE confirms the activities that he carried out and the commissioning step continues. Once the commissioning step is finished, the commissioning status of the component switches to 'waiting'. The cycle ends with documentation of the commissioning step. It includes the component's identifier, a time stamp and the actions carried out. The documentation is automatically stored in a txt-file.

Figure 7 shows the simplified sequence diagram of a commissioning step to adjust the cycle time of the pneumatic drive.

The PnP-module orchestrates the process as

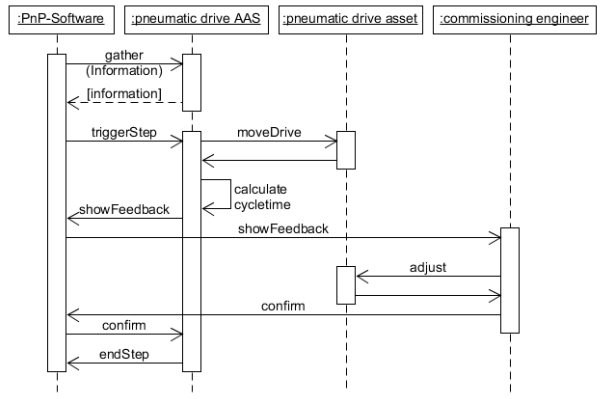

Figure 7: Sequence diagram of a commissioning step

shown in Figure 5 and interacts with the pneumatic drive, its AAS and the CE. The CE carries out adjustments at the components according to the instructions displayed on the HMI of the PnP-module. Since the AAS is deployed on the PLC, it can directly execute functions like a movement of the drive. At the same time, a function determines the cycle time from the real-time data. The opening or closing of the throttle check-valve is determined based on the deviation of the current vs. the target cycle time and gets displayed for the CE. He makes the adjustment and confirms it via the HMI afterwards. This routine is repeated continuously until the cycle time is set correctly. At this point, the commissioning step is completed and the subsequent step is selected by the PnP-module. This process is repeated as well until the system archives the final commissioning state and goes into operational mode. 


\section{VALIDATION}

The implementation and validation of the presented concept at this early stage have been successfully conducted on the demonstrator and can be summarized as follows. The introduced information model represent a key element to shift the task of instructing the commissioning from the engineer towards an automated information system. This sets the basis for further steps towards the automation of the entire process. In the current configuration of the demonstrator, improvements towards PnP have already been achieved.

First, the structuring of all commissioning tasks have been improved. No important commissioning steps have been forgotten, nor have been executed at the wrong time. Those faults happened before to an inexperienced commissioning engineer. The improvement with regard to the structure of the process, also increased the safety and repeatability of the commissioning and made it less dependent on the skills of the commissioning engineer. Second, all required information and parameters have been correctly and automatically provided to the system and engineer. In comparison to the former procedure, the engineer had to look up values, i.e. in the data sheets or the stakeholder requirements. This was a time consuming and error-prone process.

Some commissioning steps have already been automated, i.e. checking for leakage and mapping the valve spool configurations. Some steps still require manual effort, i.e. adjusting the enddamping or the throttle-check-valves. In those cases, additional actuators are required to automatically adjust the screws of the orifices.

Finally, a robust error handling hasn't been implemented up to this point. If unforeseen events or errors occur, the commissioning engineer gets a message and needs to solve the problem itself.

\section{CONCLUSION AND OUTLOOK}

In this contribution, basic enhancements of a classical pneumatic handling system towards an IIoT system are presented. The automated commissioning of this system is focused and serves as a use-case to demonstrate relevant elements of an IIoT system. A flexible and automated orchestration of different components for the commissioning requires the concept of a virtual representation of the assets, represented by the AASs. AASs have been detailed for the pneumatic system components and been implemented on the demonstrator to proof the concept. The proof-of-concept reveals that the concepts of IIoT systems can significantly structure and support the processes during the commissioning. Although it must be mentioned that the pneumatic system still relies on a commissioning engineer, since some commissioning activities can only be automated if the required functions or additional actuators are added to the system.

Consequently, developing and equipping systems with additional functionalities will be a further step for researchers and industrial manufacturers in the fluid power sector.

Additionally to approach the architecture of a distributed system, the centralized PnP-module, as presented in this paper, will further be deployed distributed on every AAS.

As a starting point, the presented concept and the information model represent a valid proof-ofconcept. But it is still in an ongoing process of iterative changes and adaptions. To have an impact on daily industrial applications the concepts needs to be further enhanced and detailed to fulfill various requirements set by different kinds of stake holders.

\section{NOMENCLATURE}

$A A S \quad$ Asset Administration Shell

$C E \quad$ Commissioning Engineer

HMI Human Machine Interface

I4.0 Industry 4.0

ICT Information and Communication Technology

IIoT Industrial Internet of Things

IoT Internet of Things

OPC UA Open Platform Communications Unified

$\begin{array}{ll} & \text { Architecture } \\ \text { PLC } & \text { Programmable Logic controller } \\ \text { PnP } & \text { Plug-and-Produce } \\ \text { SOA } & \text { Service-oriented architecture }\end{array}$

\section{ACKNOWLEDGMENTS}

This contribution results from the project 'proofof interoperability of IIoT fluid power components by the example of plug-andproduce', which is funded by the Forschungskuratorium Maschinenbau of the VDMA. 


\section{REFERENCES}

[1] Kagermann H., Wolf-Dieter L., Wahlster W. (2011) Industrie 4.0: Mit dem Internet der Dinge auf dem Weg zur vierten industriellen Revolution, VDI Nachrichten (Nr. 13), 01.04.2011

[2] Abele E., Bauernhans1 T., Reinhart G., Schuh G., (2016) WGP-Standpunkt Industrie 4.0, 2016

[3] Alt R., Schmitz K. (2019) Basic requirements for Plug-and-Produce of I4.0 fluid power systems, Fluid Power and Motion Control FPM 2019, Wuhan

[4] Schleipen M., Lüder A., Sauer O., Flatt H. Jasperneite J. (2015) Requirements and concept for Plug-and-Work, at Automatisierungstechnik 63, Heft 10, 2015

[5] Alt R., Mahlzahn J., Murrenhoff H., Schmitz K. (2018) A Survey of Industrial Internet of Things in the field of Fluid Power - basic concept and requirements for Plug-and-Produce, Proceedings of the BATH/ASME 2018 Symposium on Fluid Power and Motion Control - FPMC 2018, September 12-14, 2018, Bath, UK

[6] Piller F. (2004) Mass Customization: Reflections on the State of the Concept, The
International Journal of Flexible Manufacturing Systems, Volume 16, Springer, October 2004

[7] Dürkop L.; Jaspernite J. (2017) „Plug \& Produce" Anwendungsfall von Industrie 4.0, Handbuch Industrie 4.0 Bd. 2

[8] Alt R., Murrenhoff H., Schmitz K. (2018) A survey of "Industrie 4.0" in the field of Fluid Power, The 11th International Fluid Power Conference, 11. IFK, 2018

[9] Adolphs, P. et al (2016) Structure of the Administration Shell - Continuation of the Development of the Reference Model for the Industrie 4.0 Component, Federal Ministry for Economic Affairs and Energy, Berlin, Germany

[10] Barnstedt E. et al (2018) Details of the Asset Administration Shell: Specification, Federal Ministry for Economic Affairs and Energy, Berlin, Germany

[11] VDI / VDE / ZVEI (2016) Fortentwicklung des Referenzmodells für die Industrie 4.0 Komponente Struktur der Verwaltungsschale, Conclusionpaper

[12] Bedenbender H., Bock J., Boss B. et al (2019) Verwaltungsschale in der Praxis Wie definiere ich Teilmodelle, beispielhafte Teilmodelle und Interaktion zwischen Verwaltungsschalen. Bundesministerium für Wirtschaft und Energie 